Internist 2010 $\cdot 51: 1347$

DOI 10.1007/s00108-010-2746-7

Online publiziert: 14. Oktober 2010

๑) Springer-Verlag 2010

\author{
M. Hallek ${ }^{1} \cdot$ M.P. Manns ${ }^{2}$ \\ ${ }^{1}$ Klinik I für Innere Medizin, $\mathrm{ClO}$ Centrum für Integrierte \\ Onkologie Köln Bonn, Universitätsklinikum Köln \\ ${ }^{2}$ Klinik für Gastroenterologie, Hepatologie und Endokrinologie, \\ Medizinische Hochschule Hannover
}

\title{
Multimodale Therapie ausgewählter Tumorerkrankungen
}

Die Onkologie gehört zu den dynamischsten Feldern der Medizin und der Wissenschaft überhaupt. In kaum einen anderen Bereich der Medizin werden derzeit so viele öffentliche und private Mittel investiert. Die Onkologie gehört zweifelsohne auch zu den Feldern der Wissenschaft, in denen in den letzten Jahrzehnten durch die molekularbiologische Forschung ein erheblicher Zuwachs an pathogenetischem Verständnis erzielt wurde. Jetzt gilt es vor allem, dieses Wissen in klinisch relevante Behandlungskonzepte zu übersetzen. Dies hat ein neues Forschungsgebiet begründet, das als „translationale Forschung" bezeichnet wird. Auf diesem Gebiet ist der Forschungsbedarf besonders groß. Es gilt, die zahlreichen molekularen Therapieverfahren (sog. „targeted therapies") und die verschiedenen monoklonalen Antikörper auch wirklich zielgenau einzusetzen.

\section{> Molekulare Diagnostik kommt vor molekularer Therapie}

In den kommenden Jahren werden daher immer mehr Krebsarten erst nach ausführlicher Analyse der zugrunde liegenden molekularen Störung behandelt werden können. Diese Entwicklung hat am eindrucksvollsten bei den nicht-kleinzelligen Lungenkarzinomen begonnen, wo heute Mutationen des Epidermal-
Growth-Factor- (EGF-) Rezeptors nachgewiesen werden sollten, bevor man mit der Behandlung durch EGF-Rezeptorinhibitoren beginnt. Vor der molekularen Therapie steht also die molekulare Diagnostik. Dies erfordert das enge Zusammenarbeiten von Molekularbiologen, $\mathrm{Pa}$ thologen, Radiologen und Internisten.

Bei den meisten Tumorerkrankungen haben sich inzwischen multimodale Therapiekonzepte durchgesetzt. Viele Fachgebiete und -ärzte müssen sich abstimmen, bevor es zur Therapie eines Tumorpatienten kommt. Tumor Boards sind heute an den onkologischen Zentren eine feste Einrichtung. Damit ist die Behandlung von Krebskranken mehr denn je ein interdisziplinäres Gebiet.

In diesem Schwerpunktheft von Der Internist werden die multimodalen Therapiekonzepte von 6 ausgewählten Tumorerkrankungen beispielhaft dargestellt. Alle diese Tumorerkrankungen wie das Mammakarzinom, das kolorektale Karzinom, das hepatozelluläre Karzinom oder die Hodentumoren stellen gute Beispiele für die Bedeutung multimodaler Therapiekonzepte in der modernen Krebstherapie dar. Sie demonstrieren die Fortschritte, welche durch den Einsatz moderner Antikörpertherapien und „targeted therapies" erreicht wurden. Wir haben für dieses Heft namhafte Autoren gewinnen können, welche die aus unserer Sicht besonders bedeutsamen Fortschritte und multimodalen Therapiekonzepte häufiger Krebserkrankungen beispielhaft erläutern - im Sinne des Konzeptes eines „Fortgeschriebenen Handbuchs der Inneren Medizin“, welches Der Internist darstellt.

Es würde uns freuen, wenn dieses $\mathrm{Heft}$ das Interesse unserer Leser fände.

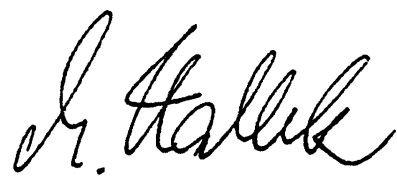

M. Hallek, Köln

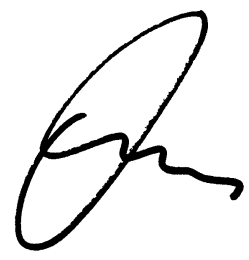

M.P. Manns, Hannover

\section{Korrespondenzadresse \\ Prof. Dr. M.P. Manns}

Klinik für Gastroenterologie, Hepatologie und Endokrinologie, Medizinische

Hochschule Hannover

Carl-Neuberg-Straße 1, 30623 Hannover

Deutschland

Manns.Michael@MH-Hannover.de 